\title{
On Representational Capacities, with an Application to General Relativity
}

\author{
Samuel C. Fletcher* \\ Department of Philosophy \\ University of Minnesota, Twin Cities \\ $\mathcal{E}$ \\ Munich Center for Mathematical Philosophy \\ Ludwig Maximilian University of Munich
}

February 14, 2018

\begin{abstract}
Recent work on the hole argument in general relativity by Weatherall (2016b) has drawn attention to the neglected concept of (mathematical) models' representational capacities. I argue for several theses about the structure of these capacities, including that they should be understood not as many-to-one relations from models to the world, but in general as many-tomany relations constrained by the models' isomorphisms. I then compare these ideas with a recent argument by Belot (2017) for the claim that some isometries "generate new possibilities" in general relativity. Philosophical orthodoxy, by contrast, denies this. Properly understanding the role of representational capacities, I argue, reveals how Belot's rejection of orthodoxy does not go far enough, and makes better sense of our practices in theorizing about spacetime.
\end{abstract}

\section{Introduction}

It is difficult to overstate just how influential Einstein's hole argument, as revived by John Stachel (1989), John Earman and John Norton (1987), has been within the foundations of spacetime theories in the last three decades. ${ }^{1}$ The argument asks us to consider two isometric relativistic spacetimes, $(M, g)$ and $(M, \tilde{g})$, such that the diffeomorphism $\psi: M \rightarrow M$ giving rise to the witnessing

${ }^{*}$ I would like to thank Gordon Belot, Neil Dewar, Ben Feintzeig, Jim Weatherall, and an anonymous referee for encouraging comments on a previous draft of this essay, which was written in part with the support from a Marie Curie Fellowship (PIIF-GA-2013-628533).

${ }^{1}$ See also Earman (1989). For reviews of the vast literature on the subject, from a range of philosophical and physical perspectives, including its bearing on broader debates about the metaphysics of spacetime, see Pooley (2013), Stachel (2014), Norton (2015), and references therein. 
isometry is the identity outside of an open set (the "hole") $O \subset M$ with compact closure. A proponent of manifold substantivalism-roughly, the view that the events of spacetime have an existence independent of their material contents - must maintain that $(M, g)$ and $(M, \tilde{g})$ are distinct because in general they assign different metrical values to points $p \in O$. Yet the laws of general relativity do not, from any proper initial data hypersurface outside of $O$, uniquely determine whether $(M, g)$ or $(M, \tilde{g})$ develops. Thus, the argument concludes, the manifold substantivalist is committed to an untoward and pernicious form of indeterminism.

Besides revitalizing discussions of the metaphysics and ontology of spacetime and determinism, the argumentative strategies used in the hole argument have strongly influenced a generation of literature on spacetime structure, the analysis of gauge equivalence and symmetry, the constraints on theories of quantum gravity, and much else (Norton, 2015, §10). On its face, the argument presents a dilemma between manifold substantivalism and determinism. Earman and Norton urge the rejection of the first horn by accepting what they call "Leibniz Equivalence," the thesis that "diffeomorphic models are equivalent" (1987, p. 522), i.e., "they represent the same physical systems" (Norton, 2015, §5). While responses to the argument have been quite varied, essentially all of them have been metaphysical, either denying some of the interpretational assumptions about general relativity needed in order to formulate the argument's dilemma, or explaining how a particular horn of the dilemma is metaphysically acceptable. ${ }^{2}$ This in turn has led many to propose modifications of the formalism of general relativity to better reflect these changed interpretational stances (Earman, 1986, 1989; Iftime and Stachel, 2006). ${ }^{3}$

Weatherall (2016b) has recently challenged this seeming consensus that a metaphysical response is truly needed to the hole argument. He argues that the dilemma can be blocked merely through more careful attention to how the mathematical structure of models used in a physical theory constrains their capacity to represent. In particular,

isomorphic mathematical models in physics should be taken to have the same representational capacities. [I.e.,] if a particular mathematical model may be used to represent a given physical situation, then any isomorphic model may be used to represent that situation equally well. (Weatherall, 2016b, p. 4)

It follows that if one commits to representing relativistic spacetimes as Lorentzian manifolds, whose isomorphisms are isometries, then there can be no ambiguity-hence no indeterminismregarding which metric field values are assigned in the "hole," for the isometry witnessing the isomorphism (which neither is nor gives rise to the identity map on $M$ ) provides the relevant standard of comparison: the value of $g$ at $p \in O$ is precisely the value of $\tilde{g}$ at $\psi(p)$. Insofar as adopting this methodological thesis blocks the hole argument, it also implies that one is not dialectically forced to modify the formalism of general relativity in response to it.

Although I agree with Weatherall's response to the hole argument, my goal here is not to provide an independent defense of it. Rather, I take it as a starting point and aim to explore his thesis regarding the concept of models' representational capacities and its interaction with mathematical equivalence (section 2), arguing in sections 3-5 for several theses regarding senses in which the mathematical equivalence of models in physical theories does constrain these capacities, and

\footnotetext{
${ }^{2}$ Exceptions include Mundy (1992) and Leeds (1995), to whose syntactic or formal responses Rynasiewicz (1996) has critically replied.

${ }^{3}$ See Rynasiewicz (1992) and Rosenstock et al. (2015) for critical discussion of Earman's proposal for Leibniz/Einstein algebras as one such formal replacement.
} 
senses in which is does not. ${ }^{4}$ I then compare these ideas with a recent claim by Belot (2017) that some isometries - generalized Leibnizian shifts- "generate new possibilities" in general relativity. Properly understanding the role of representational capacities, I argue in section 7, reveals what is correct and what is misleading in Belot's argument, and makes better sense of our practices in theorizing about spacetime. Analogously with the main argument in Weatherall (2016b), these conclusions can be drawn directly from methodological constraints on modeling - in particular, about representation - and do not force one to adopt metaphysical assumptions about spacetime. Before doing so, I thus present an intermezzo in section 6 on methodology in philosophy of physics that anticipates a possible objection to this strategy in Belot (2017). Finally, I outline the conclusions of my arguments and directions for further research in section 8.

\section{Representational Capacities and Mathematical Equivalence}

The representational capacities of a scientific model are the states of affairs that that model may be used to represent well. ${ }^{5}$ Depending on one's account of the ontology of scientific representation and representational accuracy-for instance, how representations can be inaccurate yet not nonrepresentations (Frigg and Nguyen, 2016, §1) - these representational capacities could come in degree and might be relative to a community of users of the scientific model (as is argued, e.g., by Suárez $(2003,2004))$. In investigating how mathematical equivalence of models constrains representational capacities, though, one can largely avoid committing to an account of representational accuracy by focusing on structural questions about that constraint. For example: If one model may be used to represent a state of affairs, when may another model be used to represent it equally well? If two models have the same representational capacities, how many distinct states of affairs may they represent equally well? Etc. As my arguments in the rest of this section demonstrate, one can show how equivalence of models constrains representational capacities regardless of one's account of representational accuracy, as long as that account allows for the possibility of making abstracted models more representationally accurate by adding new properties or relations to the model. ${ }^{6}$ This is a very weak requirement, arguably satisfied by all viable accounts of representational accuracy.

Similarly, for my purposes here I remain largely agnostic about most of the other major problems of scientific representations, such as whether there is a distinction between scientific and other kinds of representations, and the ontology of scientific representations. ${ }^{7}$ I only assume that scientific representation is not "radically naturalistic" (Suárez, 2003, p. 225) in the sense that "whether or not representation obtains depends on facts about the world and does not in any way answer to the personal purposes, views or interests of enquirers" (Suárez, 2003, pp. 226-7). In particular, I take for granted that the representational capacities of a mathematical model depend not just on the particular set-theoretic object that constitutes it, but also how its users consider or intend it to be part of a larger class-e.g., that a particular spacetime model represents spacetime as a Lorentzian manifold. Most major proposals for theories of scientific representation are not radically naturalistic in this sense.

\footnotetext{
${ }^{4}$ While my arguments essentially use examples only from spacetime theories, I optimistically expect the same theses to hold for physical theories generally and even any scientific theory sufficiently formalized.

${ }^{5}$ Cf. Weatherall (2016b, p. 4).

${ }^{6}$ This sense of abstraction is sometimes also known as Aristotelian idealization (Frigg and Hartmann, 2017).

${ }^{7}$ For more on these debates, see, e.g., Frigg and Nguyen (2016) and Boesch (2017).
} 
This assumption about the relevance of the intentions of the community of users of a scientific model also plays an important role in how I shall understand mathematical equivalence. Two mathematical objects, considered as members of a certain class, are deemed equivalent when they are judged to be relevantly the same as members of that class. ${ }^{8}$ Typically, that class can be described as a category, and the relevant notion of sameness is just isomorphism in that category. ${ }^{9}$ For example, isomorphism in the category of Lorentzian manifolds is just isometry, so two Lorentzian manifolds $(M, g)$ and $\left(M^{\prime}, g^{\prime}\right)$ are equivalent when they are isometric, i.e., there exists a diffeomorphism $\psi: M \rightarrow M^{\prime}$ whose pushforward $\psi_{*}$ is such that $g_{\mid \psi(p)}^{\prime}=\psi_{*}\left(g_{\mid p}\right)$ at every $p \in M$ (O'Neill, 1983 , pp. 58, 90). This commitment to a kind of mathematical structuralism (which does not entail a commitment to "philosophical" structuralism for mathematics (Awodey, 1996)) is justified by the broader mathematics community's intentions and purposes. ${ }^{10}$ That is, according to the relevant expert community of mathematicians, to commit to use a mathematical model as a member of a specific category for some purpose is to consider all isomorphic models to be equivalent for that purpose.

Having made these commitments about representational capacities and mathematical equivalence of models, I can state the three primary theses I will discuss in the next three sections. Each of them connects mathematical features of models with considerations about their ability to represent - they thus are neither purely mathematical nor purely methodological. In particular, I shall argue against the following two theses in sections 3 and 4, respectively:

Representational Uniqueness by Mathematical Equivalence (RUME) If two models of a physical theory are mathematically equivalent, then there is a unique physical state of affairs that they represent equally well.

Representational Distinctness by Mathematical Inequivalence (RDMI) If two models of a physical theory are not mathematically equivalent, then it's not the case that there is a unique physical state of affairs that they represent equally well.

The main argument against RUME and RDMI turns on the observation that the models of physical theories are abstracted, so that one can represent more features of states of affairs and relations between them by adding structure to models or relations between models. This often results in breaking the mathematical equivalence between some models.

Commitments to RUME and RDMI seem to be implicit in much of the literature on symmetry and spacetime metaphysics, but it is possible to find explicit avowals of various sorts. For example, Baker (2010, p. 1161) writes that "a physical spacetime should be given by a diffeomorphism equivalence class of mathematical spacetimes." Here, by "diffeomorphism" Baker means to indicate spacetime model isomorphisms and so accedes to both RUME and RDMI by identifying all and only the isomorphic spacetimes to represent the same physical state of affairs.

\footnotetext{
${ }^{8}$ This is not the occasion for an analysis of the "representation-as" relation (Frigg and Nguyen, 2016, §7), since the details thereof should not matter for the use to which I shall put it.

${ }^{9} \mathrm{~A}$ notable exception is the relevant notion of sameness for categories themselves, which is typically the weaker concept of categorical equivalence rather than categorical isomorphism. For more on category theory and the notions of isomorphism and equivalence therein, see, in order of increasing sophistication, Lawvere and Schanuel (2009), Awodey (2010), and Mac Lane (1998).

${ }^{10} \mathrm{Cf}$. Weatherall (2016b, pp. 3-4). The intended contrast with these intentions and purposes is with the (possibly careless or misleading) statements of individual actors. This distinction plays an important role in section 6.
} 
Earman also commits to RUME and RDMI, conditional on his preferred metaphysical solution to the hole argument.

On behalf of the relationist I propose that two [spacetime] models should be counted as equivalent (Leibniz-equivalent I shall say) just in case they can be matched up by a generalized Leibniz-shift operation; ... [such models] are different modes of presentation of the same state of affairs; that is, at base, physical states are what underlie a Leibniz-equivalence class of absolutist models. (Earman, 1989, p. 171)

A generalized Leibniz shift is just a diffeomorphism of the spacetime manifold and the pushforwards of the geometric objects defined on that manifold. RUME and RDMI then follow from the biconditional ("just in case"). Analogously, Norton (2015) writes ${ }^{11}$ that "If two distributions of fields are related by a smooth transformation, then they represent the same physical systems. ...They are merely different mathematical descriptions of the same physical reality and so should agree on all observables." This is a conditional commitment to RUME, but is agnostic on RDMI.

One can also find authors who accept RUME but deny RDMI:

[T]he right interpretation of such a theory is one in which isomorphic models of the putative semantics are equivalent (i.e., correspond to a single element of the genuine semantics). ...[W]e should not identify possible worlds with models, but rather with equivalence classes of such models under isomorphism. ... This is consistent with the claim that some non-isomorphic models are equivalent: isomorphism of models is sufficient for equivalence, but not necessary .... ${ }^{12}$ (Dewar, 2016, pp. 31-2)

Here, Dewar is mindful of the possibility that models may be distinct in a category merely because they differ on structure that one does not take to be representationally significant. ${ }^{13}$

Although I shall argue that these assertions are mistaken, perspicuous observations do lie behind them. Thus I shall argue for Weatherall's thesis, as described in section 1, which is the following weakening of RUME:

Representational Equivalence by Mathematical Equivalence (REME) If two models of a physical theory are mathematically equivalent, then they have the same representational capacities.

The main argument for REME consists in careful application of one's commitments to represent a mathematical model as an element of a certain category.

Figure 1 depicts the relationships between representational capacities and mathematical equivalence under the these positions-RUME and RDMI, on the one hand, and REME, on the other.

\footnotetext{
${ }^{11}$ See also Earman and Norton (1987, p. 522).

${ }^{12}$ Dewar is restricting attention in this statement to theories with a first-order logical formulation, but this conditional assertion of RUME and denial of RDMI is enough for my illustrative purposes.

${ }^{13}$ See also Dewar (2015).
} 


\section{Mathematical Models States of Affairs $\quad$ Mathematical Models States of Affairs}
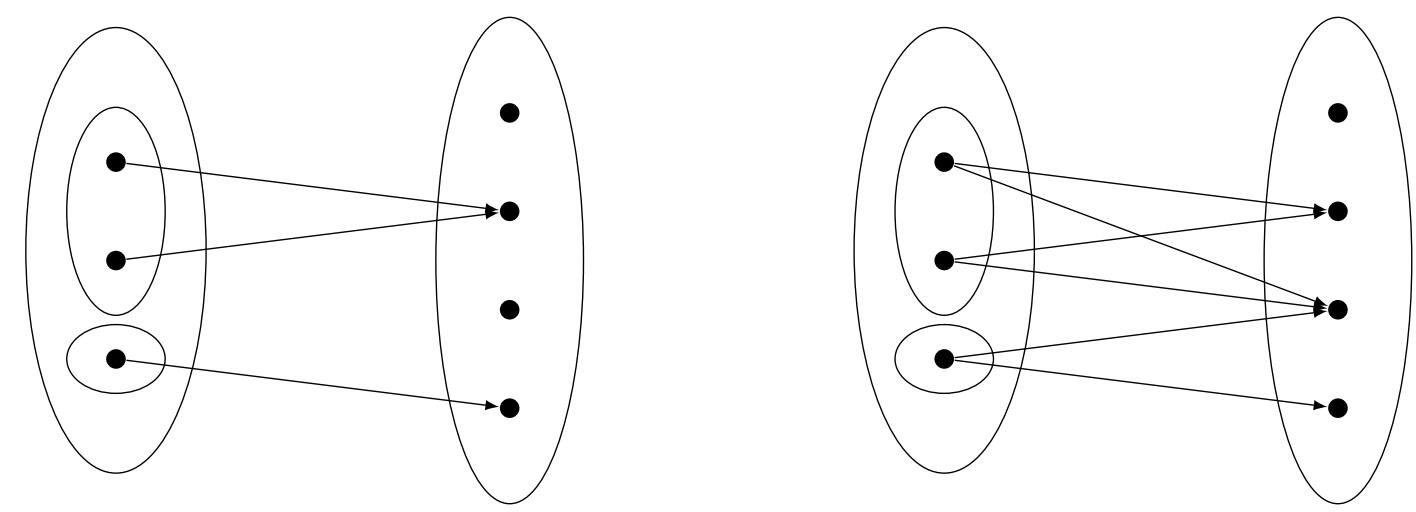

(a) Representational capacities under RUME and (b) Representational capacities under REME only. RDMI. Representation has the structure of a injective Representation only has the structure of a relation bemap on mathematical equivalence classes to states of tween mathematical equivalence classes and states of affairs.

affairs.

Figure 1: Examples of the relationships between representational capacities and mathematical equivalence. On the left, dots and the ellipses immediately around them represent, respectively, mathematical models and their equivalence classes. Dots on the right represent states of affairs. The arrows are representation relations.

\section{Against Representational Uniqueness by Mathematical Equiv- alence}

\subsection{A Warm-up: Simple Harmonic Oscillators}

Consider a block of mass $m$ sliding frictionlessly on a flat surface and connected to a rigid wall by a Hookean spring with spring constant $k$. By combining Newton's and Hooke's laws, we arrive at a differential equation for its position $x(t)$,

$$
\frac{d^{2}}{d t^{2}} x(t)+\frac{k}{m} x(t)=0
$$

whose unique solution for initial conditions $x(0)=x_{0}$ and $(d x / d t)(0)=0$ is

$$
x(t)=x_{0} \cos \left(\sqrt{\frac{k}{m}} t\right) .
$$

Consider as well an $L C$-circuit consisting of an ideal inductor, with inductance $L$, and an ideal capacitor, with capacitance $C$, connected in a loop. By Kirchhoff's laws, we arrive at a differential equation for its current $I(t)$,

$$
\frac{d^{2}}{d t^{2}} I(t)+\frac{1}{L C} x(t)=0
$$


whose unique solution for initial conditions $I(0)=I_{0}$ and $(d I / d t)(0)=0$ is

$$
I(t)=I_{0} \cos \left(\sqrt{\frac{1}{L C}} t\right) .
$$

The mathematical solutions ( 2 and 4 ) to these equations ( 1 and 3 ) are not just isomorphic as real functions of a single variable, but identical, as is evident once we perform the substitutions

$$
\left(x(t), x_{0}, m / k\right) \leftrightarrow\left(I(t), I_{0}, L C\right) .
$$

Yet they do not represent the same physical states of affairs: equation 2 represents the sinusoidal displacement of the block from equilibrium, while equation 4 represents the sinusoidal current in the circuit. The mathematical models for each abstract away all sorts of physical properties and relations particular to these two kinds of systems, the inclusion of which in the model would break their mathematical identity.

This is hardly surprising. Indeed, there is no dispute over whether isomorphic (or even identical) mathematical models can represent different physical states of affairs for different theories. But, I shall argue that similar reasoning can be employed to undermine RUME as applied within a single physical theory. In particular, I shall describe three ways in which RUME fails. First, the mathematical models of a physical theory do not determine the units of the quantities to which the models can refer. Second, these models are almost always idealized. Their abstraction or distortion allows isomorphic (indeed, the same) models to represent imperfectly distinct states of affairs equally well. I'll illustrate both of these in section 3.2 using the Schwarzschild spacetimes from general relativity. Third, RUME must fail for any theory that makes certain non-trivial modal relational claims. In section 3.3, I'll present an example of a jointed worldline, adapted from Belot (2017), that eventually "swerves" in some direction. Simply put, the modal relational claim is that the worldline could have swerved in a different direction.

\subsection{Units in and Multiplicity of Schwarzschild Spacetimes}

Consider a Schwarzschild spacetime in general relativity, whose metric line element outside its Schwarzschild radius $r_{S}$ - the location of the spacetime's event horizon-can be expressed in Schwarzschild coordinates $(t, r, \theta, \phi)$ as

$$
c^{2} d s^{2}=\left(1-\frac{r_{S}}{r}\right) c^{2} d t^{2}-\left(1-\frac{r_{S}}{r}\right)^{-1} d r^{2}-r^{2} d \Omega^{2}
$$

where $d \Omega^{2}=d \theta^{2}+\sin ^{2} \theta d \phi^{2}$ is the two-sphere line element. Schwarzschild spacetimes thus form a one-parameter family, indexed by $r_{S} \in(0, \infty)$. Now, considered as mathematical objects, the coordinates of the line element are merely numbers, and do not distinguish between meters and kilometers, seconds and minutes. So the mathematical model given by $r_{S}=1$, say, could represent the spacetime with Schwarzschild radius of one meter, one kilometer, etc. (One need only choose temporal units to keep the numerical value of $c$, the speed of light, constant.) Indeed, each mathematical Schwarzschild spacetime can represent any physical Schwarzschild spacetime (that is, with any Schwarzschild radius) through an appropriate choice of units. Consequently, RUME fails: isomorphic (indeed, identical) mathematical models can represent physically distinct states of affairs. 
A possible objection to this example is that it is a misapplication of general relativity, each of whose models (perhaps implicitly) should be distinguished by a specific choice of units. Seemingly identical models are only isomorphic when their dimensional units are also the same. According to this objection, then, when equation 5 is given, the mathematical model for an (external) Schwarzschild spacetime was not completely specified because the units of the dimensional numbers were not indicated. Once these are in fact indicated, it would be apparent that the two mathematical models were not isomorphic.

There are several responses to this objection. Firstly, one may appeal to the assumption of the normative relevance of the intentions of the community of users of general relativity: it simply isn't the case that, when they specify a relativistic spacetime, they intend to indicate the specific units in which the dimensional quantities it invokes are expressed. The mathematical models resulting from this additional specification add further structure to those of general relativity (Lorentzian manifolds). Secondly, even if one were to grant this addition, the specification of "meters" or "kilometers" as elements of the mathematical model can still be interpreted as representing, respectively, kilometers or meters. Just as with the original example, each mathematical Schwarzschild spacetime with units added can represent any physical Schwarzschild spacetime.

Another objection to this example might be advanced from the literature on the metaphysics of quantities. Motivated perhaps by relationism (Barbour, 2000), one might insist that physical quantities such as distance should be understood as grounded in or determined in virtue of their comparative relationships (Dasgupta, 2013) such as proportions (Eddon, 2013). Then in fact all the mathematical Schwarzschild spacetimes would represent a single physical state of affairs, for all the relational distance facts in each of the models are the same.

Like with the previous objection, though, this just changes the subject. Users of general relativity, such as astrophysicists, clearly intend to allow for physical Schwarzschild spacetimes with different Schwarzschild radii when they describe the parameters of various known black holes. Furthermore, even if relationism really is a superior metaphysical theory about distance properties, it is not forced upon any interpretation of general relativity according to the standards of its users.

Another way in which isomorphic (or even identical) mathematical spacetimes need not represent a unique physical spacetime is through abstraction or idealization (Lehmkuhl, 2017). To illustrate this, take again the example of a Schwarzschild spacetime. Suppose that an astrophysicist is interested in modeling, say, a binary black hole merger, in which two black holes of the same Schwarzschild radius coalesce into one. Much before the collision, each of the two black holes may be modeled as its own mathematical Schwarzschild spacetime-perhaps truncated at large distances from the horizon-with the same Schwarzschild radius. Despite their mathematical models being identical, the physical states of affairs they represent are distinct, for otherwise no collision could occur! In contrast with, say, a universe containing only two qualitatively identical iron spheres (Black, 1952), the astrophysicist does not intend her model to represent a universe empty of everything except two qualitatively identical black holes. This is possible in part because other features that might distinguish the two black holes, e.g., their positions relative to each other and other matter, have been abstracted away - thus the aptness of describing the representational targets of mathematical models "states of affairs" rather than possible worlds.

This feature invites the objection that RUME should apply when the mathematical models represent not just (proper) states of affairs, but whole possible worlds. However, such a restriction is ad hoc, and indeed nearly all the mathematical models used by scientific theories are not intended as representing whole possible worlds. If modern physical cosmology is an exception to this, 
it does not mean that the application of general relativity is restricted to the whole universe, as the astrophysicist's trade exhibits. ${ }^{14}$ Even when general relativity is applied to cosmology, its models are still idealized, abstracting or distorting features so that quite different universes can be represented with the same mathematical model.

\subsection{A Jointed Wordline: Directions to Swerve}

Consider Minkowski spacetimes with a distinguished worldline representing, say, the history of a particle of interest. At first the particle is unaccelerated, i.e., its worldline is geodesic, and then at some event it begins to accelerate at a constant rate in a particular direction. In standard coordinates $(t, x, y, z)$ of the frame of reference determined by the particle while it moves inertially, its worldline could be given by

$$
\begin{aligned}
& t(\tau)= \begin{cases}\tau & \text { if } \tau \leq 0, \\
(c / a) \sinh (a \tau / c) & \text { if } \tau>0,\end{cases} \\
& x(\tau)= \begin{cases}1 / a & \text { if } \tau \leq 0, \\
\left(c^{2} / a\right) \cosh (a \tau / c) & \text { if } \tau>0,\end{cases} \\
& y(\tau)=0, \\
& z(\tau)=0,
\end{aligned}
$$

where $\tau$ is a parameterization of the particle's proper time and $a$ is its constant (proper) acceleration after $\tau=0$.

In fact, the details of the worldline are not so important as the fact that it begins to swerve in a particular direction at some time. ${ }^{15}$ Users of relativity theory, I claim, would intend for the theory to endorse that the particle could have swerved (at the same acceleration) in another direction, even at another time, than it did in the above model. Such alternative states of affairs could be realized by a spatial rotation or time translation acting on the above model. Yet the resulting model is in fact isomorphic to the one above-the isometry witnessing this is just that generated by the spatial rotation or time translation. Yet if RUME were true, these mathematical models would have to represent exactly the same state of affairs. Just as with the case of the Schwarzschild spacetimes, whether some sort of relationism, according to which there is only one state of affairs to represent, is metaphysically preferred is not relevant for the point at issue, which is how to represent the theory that does take there to be many such distinct states of affairs. Proposals to identify isomorphic but non-identical models in various ways (Earman, 1989; Iftime and Stachel, 2006; Baker, 2010), which are often motivated by a desire for representational uniqueness (perhaps for metaphysical ends), also must face the awkward problem of how to represent these distinct states of affairs with a single model.

\footnotetext{
${ }^{14}$ In contrast with Belot (2017), this is not to say that general relativity needs two sectors of models with different identity conditions, one of which is used for cosmology and another for localized astrophysical modeling, independently of the intentions of the users of those models. I will return to this point in section 7.

${ }^{15}$ The example is an amalgam of Wilson's buckling beam (1993) and Belot's Epicurean-fashioned swerve theory (2017), set in Minkowski spacetime.
} 


\section{Against Representational Distinctness by Mathematical Equiv- alence}

Consider again the Schwarzschild spacetime metric (equation 5) and the (proper) homothety $g_{a b} \mapsto$ $C g_{a b}$, with $C>0$ and $C \neq 1$. Let $r_{S}^{\prime}=\sqrt{C} r_{S}$, so that one can write

$$
\begin{aligned}
C c^{2} d s^{2} & =\left(1-\frac{r_{S}}{r}\right) c^{2}\left(\frac{r_{S}^{\prime}}{r_{S}}\right)^{2} d t^{2}-\left(1-\frac{r_{S}}{r}\right)^{-1}\left(\frac{r_{S}^{\prime}}{r_{S}}\right)^{2} d r^{2}-\left(\frac{r_{S}^{\prime}}{r_{S}}\right)^{2} r^{2} d \Omega^{2} \\
& =\left(1-\frac{r_{S}^{\prime}}{r^{\prime}}\right) c^{2} d t^{\prime 2}-\left(1-\frac{r_{S}^{\prime}}{r^{\prime}}\right)^{-1} d r^{\prime 2}-r^{\prime 2} d \Omega^{2}
\end{aligned}
$$

by setting $r^{\prime}=\left(r_{S}^{\prime} / r_{S}\right) r$ and $t^{\prime}=\left(r_{S}^{\prime} / r_{S}\right) t$. Thus, a change of variables reveals that this homothetic spacetime has a metric which can be put into the same form as a Schwarzschild spacetime with Schwarzschild radius $r_{S}^{\prime}$. But by the arguments of section 3.2, this spacetime can in fact represent any physical Schwarzschild spacetime. It follows that each spacetime homothetic to some mathematical Schwarzschild spacetime can represent equally well each physical Schwarzschild spacetime-i.e., with any Schwarzschild radius. ${ }^{16}$ Yet the models are not isometric to one other, i.e., they are not mathematically equivalent as Lorentzian manifolds, for their Schwarzschild radii, which are coordinate-independent, are numerically distinct. Hence RDMI is false.

The core observation of the above argument is that two mathematical models of a theory may have different mathematical structures - they may fail to be isomorphic as members of their intended category - that do not make a difference to their representational capacities. Not all structures that make a mathematical difference between models need make a representational difference—cf. the "qualified realism" of Dewar (2015).

This observation also invites the objection that theories whose mathematical models have structure that does not make a difference to their representational capacities are somehow defective: they represent excess structure that ought to be eliminated-e.g., by expanding the maps which count as isomorphisms - as with the vector-potential formulation of electromagnetism (Weatherall, 2016a,c) or symmetries more generally (Dewar, 2017). Such motivations underlie a similar response to the hole argument (Earman, 1989; Iftime and Stachel, 2006; Baker, 2010). But, as with similar objections raised in the previous sections, this one just changes the subject: Even if there are in general good reasons for preferring theories without excess structures over those with it, this does not preclude the latter from representing physical states of affairs legitimately.

\footnotetext{
${ }^{16}$ Belot (2013, p. 331) suggests that homothetic spacetimes in general are "physically equivalent" for those motivated by relationism to "deny that there are possible worlds that agree about distance ratios but disagree about matters of absolute distance." However, the arguments of section 3.2 show that no such metaphysical assumption is needed for this conclusion (if one reads "physically equivalent" as "having the same representational capacities"). (A further caution: Belot describes homotheties as "scaling symmetries," but this description may be misleading when matter fields introducing their own length and time scales are present.)
} 


\section{For Representational Equivalence by Mathematical Equiva- lence}

\subsection{Intentional Commitments}

Even though (in section 3) I have argued that RUME is false, a weakened version of it, REME, should hold: mathematically equivalent models have the same representational capacities. Much of the argument for it has already been given in section 2 . When one selects a category of mathematical models with which to represent a range of physical states of affairs, one is representing those states affairs as models of that sort. Consequently, any differences, e.g., in set-theoretic construction, between mathematical models that are equivalent are representationally superfluous. To assert that one model represents a state of affairs better than another, it seems, demands some differences between the models to make the representational difference between them. But if those differences are not sufficient to make the models inequivalent, then in fact they violate the intention to represent the states of affairs as models of a certain sort. ${ }^{17}$

By analogy, consider an architectural scale model: as a scale model, it represents the physical dimensions and relative positions of the elements of an architectural design. Whether the model is constructed from pieces of metal, plastic, wood, or paper makes no difference. So if one were presented with two dimensionally equivalent scale models constructed from different materials, yet demanded that the two must represent different architectural designs, then one could not consistently be considering the models merely as scale models. Commitment to REME, simply put, follows from one's intentional commitments to represent a state of affairs as an object from a specified category. ${ }^{18}$

\subsection{Relations between and Additional Structure on Mathematical Models}

One concern that might arise about accepting REME but rejecting RUME is that it precludes a resolution of the hole argument, adumbrated in section 1, in favor of determinism. If two relativistic spacetimes, represented as Lorentzian manifolds, are related by a hole transformation, then they are isometric, i.e., related by a map that is an isomorphism in the category of Lorentzian manifolds. That means, by REME, that they have the same representational capacities, but not necessarily that they must represent the same unique physical state of affairs. Yet if this represented state of affairs is not unique, then the problem of indeterminism seems to rise again, forcing one to confront a metaphysical dilemma anew.

The error implicit in this concern is the assumption that Lorentzian manifolds, as mathematical models, represent all properties of a physical relativistic spacetime (and its "contents"). As I ar-

\footnotetext{
${ }^{17}$ Within the hole argument literature, Butterfield (1989) and Maudlin $(1989,1990)$ developed positions which are incompatible with REME, in the sense that once one has set a particular Lorentzian manifold to represent a spacetime, those related by to it by a non-identity isomorphism do not. (As I discuss in section 5.2, whether they do in fact depends on a choice of map by which to compare the two.) For discussion of these positions, including their demerits, see also illuminating discussion in Rickles (2008, Ch. 5) and Pooley (2013). Perhaps others have on other topics, but I have not canvassed the literature.

${ }^{18}$ For more arguments that could be mustered in favor of REME, see Dewar (2016, ch. 2). Although Dewar's thesis is in fact RUME, much of his argumentation could be adapted in support for REME in light of the considerations of section 3.
} 
gued in section 3, that some properties are abstracted away-such as the representation of units or additional structure, e.g., additional fields-is true here just as it is in essentially all other contexts of mathematical modeling and scientific representation. Lorentzian manifolds may not exemplify properties of the states of affairs they represent, but all the properties they do exemplify-those not abstracted away-are the same for isomorphic manifolds. This is precisely encoded in the mathematical models themselves with the interpretation of isomorphic objects in a mathematical category as being equivalent as objects in that category. And a claim of isomorphism is just an existence claim that there is a bijective map of a certain sort that preserves the structures comprising the mathematical object in question. So any putative representational differences among isormorphic models, such as spacetime point haecceities, is not reflected at all in the models themselves as members of category they are taken to be-there is no mathematical correlate of those differences definable in the category. ${ }^{19}$ Thus, the fact that the mathematical models do not determine these putative properties, rather than being surprising and substantive, is entirely expected and benign. This sort of conclusion, that the only properties left undetermined are those we should have expected were so, is not itself new in the literature, but they had previously been argued only through a commitment to a particular spacetime metaphysics such as substantivalism (Brighouse, 1994, 1997, 2008). By contrast, I have arrived at the same conclusion without any such commitments about the metaphysics of space, time, or events.

Just because the elements of a collection of mathematical models are isomorphic, however, does not mean that "equivalence" is the only interesting relationship among them. As I argued in section 3.3, one reason to reject RUME is that many distinct states of affairs ought be represented by isomorphic mathematical models. To show how this works, consider models of the swerving particle worldline from section 3.3 and the claim that the particle could have swerved in a different direction and at a different time than it did in a particular model representing it. First, we can describe the former as tuples $(M, \eta, \gamma)$, where $M$ is diffeomorphic to $\mathbb{R}^{4}, \eta$ is the Minkowski metric, and $\gamma: \mathbb{R} \rightarrow M$ is the swerving worldline. Importantly, the supplement to the usual Minkowski spacetime structure by the worldline $\gamma$ is not essential for the models of the swerve theory to represent the many different possible histories of the particle, but it does make them more representationally complete. (This addition of structure, which distinguishes the "swerve" case from the hole argument case, will play an important role in section 7.) Second, let $T_{t}: M \rightarrow M$ represent a time-translation of Minkowski spacetime by $t$ units, and $R_{\rho, \phi}: M \rightarrow M$ a rotation of angle $\phi$ about the spatial axis $\rho$ centered on the origin. Next one must pick a standard of comparison-a particular isomorphism in the category, which we will take as our standard for evaluating relations between models. ${ }^{20}$ For the sake of generality, let it be some diffeomorphism, $\psi: M \rightarrow M$, and its pushforward, $\psi_{*}$, acting on Minkowski spacetime.

Now, consider the model $\left(M, \eta,\left(T_{t} \circ R_{\rho, \phi} \circ \psi\right)[\gamma]\right)$. Because we have chosen $\psi$ as our standard of comparison, we act on the model by $\psi^{-1}$ and its pushforward, as is appropriate,

$$
\left(\psi^{-1}[M], \psi_{*}^{-1}[\eta],\left(\psi^{-1} \circ T_{t} \circ R_{\rho, \phi} \circ \psi\right)[\gamma]\right)=\left(M, \eta,\left(\psi^{-1} \circ T_{t} \circ R_{\rho, \phi} \circ \psi\right)[\gamma]\right),
$$

checking whether it is the same as $(M, \eta, \gamma)$. Evidently this occurs if and only if $t=\phi=0$. So, except in this case, $\left(M, \eta,\left(T_{t} \circ R_{\rho, \phi} \circ \psi\right)[\gamma]\right)$ represents a time translation and spatial rotation of

\footnotetext{
${ }^{19}$ Of course, this is compatible with a skeptical attitude towards such properties but in this case no problem about determinism arises.

${ }^{20}$ As Weatherall (2016b, p. 6) emphasizes, "All assertions of relation between mathematical objects-including isomorphism, identity, inclusion, and so on-are made relative to some choice of map."
} 
$(M, \eta, \gamma)$, relative to $\psi$. If, by contrast, one had used instead $T_{t} \circ R_{\rho, \phi} \circ \psi$, for some fixed $t, \rho$, and $\phi$ as the standard of comparison, then it would not represent any difference at all. So, changing which isomorphism one uses as a standard of comparison can change the relations between different models, but such changes do not affect any fact about the existence of models bearing these relations: these are invariant under isomorphism. While each model in a collection may be isomorphic to each other, the collection as a whole, with the relations that hold between them, may not be isomorphic to the singleton collection! ${ }^{21}$

Further, there is nothing representationally distinguished about any of the isomorphisms. In particular, it is often computationally convenient to chose the identity map for $\psi$, but that privilege does not extend to its representational features. Similarly, "the fact that the identity has certain mathematical properties that distinguish it from other maps does not mean that it is privileged for the purposes of characterizing relationships between mathematical objects" (Weatherall, 2016b, p. 6n).

These sorts of conclusions too- that, e.g., the swerve models do not represent exactly one state of affairs, endorsing the claim that the swerve could have happened at a different time and in a different direction-are not new in the literature. But they had heretofore required increasingly sophisticated investigations into the metaphysics and interpretation of Lewisian counterpart theory (Belot, 1995; Brighouse, 1997; Melia, 1999; Brighouse, 2008; Arntzenius, 2012; Dewar, 2016; Belot, 2017). ${ }^{22}$ By contrast, I have arrived at the same conclusion without any such commitments about the metaphysics of possible worlds or the counterpart relation holding between objects in them. ${ }^{23}$ (Although a choice of diffeomorphism $\psi$ does serve to compare models, the choice thereof is essentially conventional, as described in the previous paragraph.) Whether this undercuts some of the motivation for investigating the metaphysics of counterpart theory for spacetime theory I shall let readers decide, but it is nonetheless surprising that a metaphysical investigation can be side-stepped by a commitment to a methodological principle, REME, about how mathematical structure constrains representation.

\section{Elvis Has Left the Building}

In section 7, I will apply the foregoing consideration to the case of general relativity, comparing them with recent claims by Belot (2017) that some isometries "generate new possibilities." Before I do so, in this section I address a methodological objection to this comparison that one could reconstruct from Belot (2017).

In section 2, I described how I am assuming that the representational capacities of a mathematical model have an intentional component, in the sense that they depend on how its users intend it to be a part of a larger class. And I have taken the relevant class of users to be mathematicians and

\footnotetext{
${ }^{21}$ There is an analogy here with Muller's (2011) defense of spacetime structuralism against the charge by Wüthrich (2010) that each event of a homogeneous spacetime has the same profile of properties, so if events are discerned from one another by such properties, then there would only be one such event. Muller simply points out events may be discerned not only by their (absolute) properties but also their relations with other points.

${ }^{22}$ See also Dees (2015, ch. 5) for metaphysical arguments in parallel to mine about the representation of physical units in section 3.2.

${ }^{23}$ Cf. the position of Weatherall (2016b) vis-à-vis those of Butterfield (1989), Brighouse (1994), and Pooley (2013, $\S 7)$.
} 
mathematically grounded scientists. ${ }^{24}$ It could be objected, though, that this is the wrong class: one should look instead to the perhaps distinct methods and practices of the broader class of relativists. Any attempt to impose considerations extrinsic to that community's, whether philosophical or mathematical, would be misplaced in understanding how representation and equivalence work in general relativity, especially if the physicists outnumber philosophers and mathematicians.

Belot explicitly endorses this kratocratic account of possibility and representation when it comes to spacetime theory:

About some things - such as the fact that Elvis was peachy-keen-his legions of fans could not be wrong, just because there were so many of them. Similarly, might makes right whenever a large group of people uses a word or a concept in a certain way ...[T] here is a large community of people [physicists] according to whose modal concepts shifts generate new possibilities-large, that is relative to the number of philosophers interested in these matters. So shiftless philosophers are engaged in a revisionary project of trying to construct new modal concepts to replace ones in common use. (Belot, 2017, pp. 1, 9)

In Belot's terminology, a "shift" is an isometry of spacetime-temporal and spatial "shifts," as we find them discussed in the Leibniz-Clarke correspondence and the literature around it, are the most familiar examples. Philosophers who are "shiftless" are those who deny that isometries "generate new possibilities." In the next section I will bring attention to what this could mean exactly in the context of RUME and REME. But what is important here is Belot's methodological claim about the priority of physicists' "modal concepts" over philosophers when it comes to spacetime theory and the grounds for it—-this is why the title of Belot (2017) is "Fifty Million Elvis Fans Can't Be Wrong."

I agree with Belot that it would be likely fruitless to hold physicists to methods, standards, or ideas extrinsic to their work and goals. But one can and should engage in critique of physicists' use of "modal concepts" with methods, standards, and ideas to which the physics community is already committed. Just as Belot (2017, p. 23) emphasizes that the mathematical models of a scientific theory are not "a mere jumble" but have structure, the commitments of a community are not well captured by bare descriptions of what the plurality say or do. In the first place, one cannot "read off" these commitments literally from what they say any more than one can "read off" an interpretation of a theory from its formalism more generally (Dewar, 2015; Lehmkuhl, 2017). Neither can one do so from what they do, for this would reify blunders as well as real commitments. In the case at hand, one of those commitments is to the use of mathematical methods and concepts, as much as is possible, as they are best understood mathematically. Although physicists do not demand complete mathematical rigor, they are always receptive to concerns about the misuse of well-understood mathematics. ${ }^{25}$ For example, committing a mathematical mistake is grounds for criticism in the physics community, even if there is no explicit norm about that particular mathematical affront. In this sense, the physics community is indeed committed to representing mathematical models as members of a particular category in the same way mathematicians are.

\footnotetext{
${ }^{24} \mathrm{Cf}$. the demand that "we need to be sure that we are using the formalism correctly, consistently, and according to our best understanding of the mathematics" (Weatherall, 2016b, p. 2).

${ }^{25}$ The qualification, "well-understood," is important here, for physicists' attitude towards less understood formalism is more liberal, as the historical use of infinitesimals, Dirac delta functions, etc., attest.
} 
It is important to emphasize that this commitment is not born from or justified by mathematical considerations alone: the reasons mathematicians have adapted the commitments they have is partly through interactions with and sensitivity to applications in the physical sciences. To defuse a similar concern in the context of the account of her naturalistic metaphilosophy, Maddy (2007, pp. 349-350) describes how

One might worry that this leaves the well-being of science at the mercy of the mathematician's whim. ...Though they may not be primarily motivated by physical applications, providing tools for natural sciences remains one among the overarching goals of the practice of mathematics; ... Contemporary mathematicians-like contemporary scientists — take for granted that a tool doesn't work well for no reason; they tirelessly pursue explanation.

Moreover, scientists' adherence to mathematicians' commitments regarding, e.g., mathematical equivalence does not depend on some slavish tracking of the mathematical community, but rather the functional role that mathematical practice plays in science (Maddy, 2007, p. 351). That's to say that scientists accept these commitments about mathematical equivalence not in virtue of mathematicians' practices in doing mathematics qua mathematics, but because of the success and fruitfulness of applied mathematics, which also shapes mathematical practice.

To see how this response would apply to a different but still analogous concrete case, compare Belot's quotation above to the fictional "Jordan Benot" writing a bit before 1950 in favor of a kratocratic account of physical quantities:

There is a large community of people [physicists] according to whose principles only measurements provide meaning to physical quantities-large, that is relative to the number of philosophers interested in these matters. So realist philosophers are engaged in a revisionary project of trying to construct new principles to replace ones in common use.

This is not an ahistorical fiction, for Benot could have well been speaking about the dominance of Heisenberg's thinking on measurement in quantum mechanics:

It would be difficult to find a textbook of the period [1930-1950] which denied that the numerical value of a physical quantity has no meaning whatsoever until an observation has been performed. (Jammer, 1974, p. 246)

Benot's kratocracy about physical quantities would entail that the philosophers and wayward physicists who struggled to convince the broader physics community about the importance of the measurement problem (Freire, 2015) were at best conceptual revisionists, at worst simply wrong: Heisenberg's concept of physical quantity could not have been wrong, just because there were so many who endorsed it. By contrast, we can understand why these philosophers and physicists were justified by appeal to the commitments that the physics community already endorses and strives (however imperfectly) to fulfill: conceptual clarity and logical consistency.

There is, of course, a difference between unobserved physical quantities and physical possibilities. But the relevant point of analogy is that Heisenberg's position about unobserved quantities, as described above, entails a position about what possibilities there are and are not, namely, that there are no possible unobservered physical quantities. The nonconformist philosophers and physicists 
working on the measurement problem were not just concerned with unobservables, but all that follows from theorizing about them, including what's possible and not, according to the physics community's commitment to follow the consequences of a theory wherever they may lead. This is how fifty million Elvis fans can be wrong: by their own lights, rabid cheers for encore are no longer apt when Elvis has left the building.

\section{Belot's Two Sectors}

In his (2017), Belot presents several arguments regarding when various isomorphisms of spacetimes structures "generate new possibilities." He considers both relativistic and non-relativistic spacetimes, as well as esoterica such as Yang-Mills-Higgs solitons. In this section, I restrict attention to his claims regarding general relativity. ${ }^{26}$

Belot (2017, p. 14) notes that "very nearly everyone seems to agree that on an acceptable interpretation of general relativity, no generalized shifts generate new possibilities." (A "generalized shift" here is simply an isometry.) By contrast, he wishes to argue "that we should take some generalized shifts to generate new possibilities in general relativity" (Belot, 2017, p. 4, emph. added). In particular, Belot distinguishes between two "sectors" of general relativity, that for cosmology and that for (what can be characterized at first pass as) isolated systems. These divide the spacetime models of general relativity by the (typical) intention with which they are used by relativists. The first includes only relativistic spacetimes that are intended as models of the universe. These are the ones to which the consensus about generalized shifts applies: spacetimes are isometric here if and only if they are "gauge equivalent," meaning that "they have to be viewed as representing the same possible situation (because they jointly represent just one possibility)" (Belot, 2017, pp. 15-16).

But this is not so for isolated systems. They are modeled by spacetimes that are asymptotically flat (at spatial infinity), whose models are triples $(M, g, \eta)$, where $M$ is a manifold isomorphic to $\mathbb{R}^{4}, g$ is a Lorentzian metric on $M$, and $\eta$ is a "non-physical" Minkowski metric on $M$. They satisfy the additional property

that if one goes far enough towards spatial infinity in any direction, one can find sufficiently small regions of [the manifold $M$ ] such that the geometry of such a region according to $g$ is arbitrarily similar to the geometry of that region according to $\eta$. (Belot, 2017, pp. 19-20)

For our purposes, it is not so essential to give a precise characterization (for which see Christodoulou (2008)) as to note that, for Belot,

This extra "background structure at infinity" allows us to make finer-grained distinctions between isometric solutions than we can in the cosmological case-it becomes possible, e.g., to think of two such solutions as differing by a "time translation at infinity." (Belot, 2017, p. 21)

Belot has in mind here diffeomorphisms $\phi: M \rightarrow M$ that induce an isometry of $g$ and of $\eta$, where that induced for the latter is in particular a time translation (or at least asymptotically so). Similar conclusions are supposed to hold for other elements of the Poincaré symmetry group acting on $\eta$.

\footnotetext{
${ }^{26}$ I think my conclusions drawn here also extend to Belot's claims about non-relativistic spacetimes, but I shall not argue my case here.
} 
In order to evaluate Belot's conclusions about the two sectors in light of the above arguments against RUME and for REME, one might first ask what it means for an isometry to "generate new possibilities." On this question, Belot does not give an explicit answer. From the contrast he draws between the cosmological and asymptotically flat sectors in terms of "gauge equivalence," it would at first seem that "generating new possibilities" entails the rejection of RUME. It is further natural to read it as declaring that the representational capacities of the new mathematical model-the result of the "generalized shift" - are not contained in those of the first: the shifted model can represent possibilities that the unshifted model cannot, which would be a rejection of REME as well. Yet, he writes in a footnote that he takes "for granted that isomorphic solutions are always representationally equivalent" (Belot, 2017, p. 29) and on one occasion glosses his position seemingly more weakly as being that "even in general relativity, isomorphic solutions can sometimes be capable of representing distinct possibilities" (Belot, 2017, p. 27). This all fits with the interpretation that Belot indeed rejects RUME, and what is generated by the appropriate shifts in asymptotically flat spacetimes are rather non-trivial inter-model representational relations, in the sense described in section 5.2 between models of the swerve theory: if one model is taken to represent a certain state of affairs, then another related by a non-trivial isometry would represent a distinct state of affairs.

If this interpretation is correct, then I agree with Belot's "baldly heretical" (2017, p. 1) conclusion in this case, with two important qualifications. As I described in section 5.2, whether two isomorphic models satisfy a relation of representational distinctness depends on a choice of map by which to compare the two. In implicitly using the identity map for these purposes, Belot does not acknowledge this relativity; if he had chosen differently, it would have lead to a different representational relation-perhaps even the identity, in which case no new representational relations would be generated. So, whether the relation between two isometric models is one of representational distinctness is not an absolute matter, as Belot seems to suggest, but is always relative to a choice of isomorphism by which one compares them.

Second, the use of asymptotic flatness to define a special sector in which RUME fails and isometries represent non-trivial inter-model representational relations is a bit of a red herring. It is true that physicists use general relativity to model different sorts of states of affairs, some cosmological and some astrophysical. And, as Belot (2017, pp. 22-25) points out, the relation between asymptotic structure and various types of global conserved quantities is important to characterize properties of those spacetimes such as total mass and angular momentum and their connections with analogous concepts from other parts of physics. Although it is tempting to let oneself be dazzled by the sophisticated mathematical gadgetry used in modern spacetime theory, this gadgetry isn't particularly relevant to why RUME fails: the arguments of section 3 apply whether or not a spacetime is equipped with extra structure such as a reference Minkowski metric with respect to which asymptotic flatness is defined.

Comparison with the swerve theory is helpful here. In that case, the addition of structure to a model of Minkowski spacetime $(M, \eta)$ — the distinguished worldline $\gamma: M \rightarrow M$ representing the history of the swerving particle-allows us to better represent the different ways the world could be. Relative to, say, the identity map on $M$, models related to $(M, \eta, \gamma)$ by a non-trivial temporal translation $T_{t}$ or rotation $R_{\rho, \phi}$ represent the swerve occurring at a different time or in a different direction. But if that structure were removed, yielding standard Minkowski spacetime again, one could still maintain the same conclusions about the different ways a particle worldline could swerve. For, any worldline satisfying the constraints for the swerve could represent the 
particle equally well. Minkowski spacetime $(M, \eta)$ is not as detailed of a representation as $(M, \eta, \gamma)$ because it has abstracted away exactly which points represent the particle worldline, but it can still represent the particle nonetheless. Analogously, one can represent an asymptotically flat spacetime $(M, g, \eta)$ as a spacetime $(M, g)$ without altering the conclusions drawn from it. In that case, one only needs the existence of a Minkowski metric which the actual spacetime metric $g$ approximates asymptotically; it needn't be added as a new piece of spacetime structure to do so. Although $\eta$ is not intended to represent an object as $\gamma$ is, so that they there isn't the issue of having a less detailed representation, the two cases are relevantly similar because the conclusions about what the spacetime (and its "contents") represents are the same.

This leads me to my disagreement with Belot about RUME in the cosmological case. Belot accepts what he takes to be the orthodox conclusion of the hole argument that endorses RUME when, e.g., there are no asymptotic boundary conditions like asymptotic flatness imposed. One of his principle reasons for this is that, in the latter case, there are isometries which do not entail a kind of indeterminism.

At best, the [hole] argument shows that we should deny that certain generalized shifts generate new possibilities - namely, those that involve us in indeterminism if they are not handled that way. But it does not obviously follow that we should deny that any generalized shifts generate new possibilities. (Belot, 2017, p. 14)

However, the starting point in this paper has been Weatherall's response to the hole argument, which points out that it does not force us into a metaphysical dilemma regarding a kind of indeterminism: once we have committed to representing relativistic spacetimes as Lorentzian manifolds and to REME, either there is no ambiguity regarding the spacetime metric inside the "hole," or any ambiguity that persists consists only in properties that have not been represented at all. With the specter of substantive indeterminism vanquished, the argument for adopting RUME is undermined, in consonance with my arguments against it in section 3.

\section{Conclusions and Future Work}

Although Weatherall (2016b) assessed his argument regarding the hole argument's force as largely negative, I am inclined to say that it reveals how the metaphysical cast of the literature on the hole argument has obscured the more significant considerations it raises for scientific representation. In particular, by basing his argument on commitments about how mathematical equivalence constrains representational capacities-REME, in particular-Weatherall has invited a new positive direction to the literature. Accordingly, one of my goals in this article (section 2) has been to articulate some of the theses about these sort of constraints-RUME, RDMI, and REME - that are implicitly assumed but rarely explicitly discussed in much of the literature. I argued that, contrary to many, we should reject RUME (section 3) and RDMI (section 4) in general but accept REME (section 5). The rejections arose because of the abstracted nature of mathematical models, from the referents of particular physical units for, e.g., distances and times, and from the need to represent modal relations between even isomorphic models. But our commitments to represent the states of affairs of relativistic spacetimes as Lorentzian manifolds just means that we have taken differences between Lorentzian manifolds to not make a difference in their representational capac- 
ities (REME), as befits the physics community's commitment to mathematical norms. This is in contrast with Belot's modal kratocracy (2017), which we should reject (section 6).

I also compared my conclusions with the recent proposal by Belot (2017) to consider some isometries of asymptotically flat spacetimes to "generate new possibilities." If one interprets this notion of "generation" as the production of an inter-model relation of representational distinctness, then my conclusions dovetail with Belot's, with the qualification that these relations are not absolute but always relative to a choice of comparison map. However, contra Belot, the addition of asymptotic boundary conditions is not actually relevant for this conclusion. Accordingly, his primary reasons for coming to different conclusions for the asymptotically flat sector of general relativity than for the cosmological sector no longer hold, which leads to the general rejection of RUME that I espouse.

The implications of rejecting RUME and RDMI but accepting REME need further exploration for other theories of spacetime, but also for those of matter and other scientific disciplines. For example, debates about the interpretation of unitary equivalence (Ruetsche, 2011) and broken symmetries for infinite quantum systems (Baker, 2011) hinge on similar questions regarding the relationship between mathematical equivalence and representational capacities-perhaps Feintzeig's (2015; 2016) interventions in these debates can be understood in these terms. And generally, rejecting RUME undercuts much of the motivation for proposals to quotient the models of theories (such as general relativity) by isomorphism (Earman, 1986, 1989; Iftime and Stachel, 2006), for in doing so one identifies models that might represent different states of affairs. This may suggest a new way of understanding the role and interpretation of gauge theories (Weatherall, 2016c), including answering the apparent puzzle of why their alleged use of surplus structure is so scientifically useful (Rovelli, 2014) - perhaps it is because it is representationally useful. Far from being stagnant, thinking about the hole argument in representational terms promises to yield new answers and fruitful research directions to many other lines of inquiry.

\section{References}

Arntzenius, F. (2012). Space, Time, and Stuff. Oxford University Press, Oxford.

Awodey, S. (1996). Structure in mathematics and logic: A categorical perspective. Philosophia Mathematica, 4:209-237.

Awodey, S. (2010). Category Theory. Oxford University Press, Oxford, 2nd edition.

Baker, D. J. (2010). Symmetry and the metaphysics of physics. Philosophy Compass, 5(12):11571166.

Baker, D. J. (2011). Broken symmetry and spacetime. Philosophy of Science, 78(1):128-148.

Barbour, J. (2000). The end of time: The next revolution in physics. Oxford University Press, Oxford.

Belot, G. (1995). New work for counterpart theorists: Determinism. The British Journal for the Philosophy of Science, 46(2):185-195. 
Belot, G. (2013). Symmetry and equivalence. In Batterman, R., editor, The Oxford Handbook of Philosophy of Physics, pages 318-339. Oxford University Press, Oxford.

Belot, G. (2017). Fifty million Elvis fans can't be wrong. Noûs, pages n/a-n/a.

Black, M. (1952). The identity of indiscernibles. Mind, 61(242):153-164.

Boesch, B. (2017). Scientific representation. In Zalta, E. N., editor, The Internet Encyclopedia of Philosophy. Accessed 29 August 2017.

Brighouse, C. (1994). Spacetime and holes. PSA 1994: Proceedings of the Biennial Meeting of the Philosophy of Science Association, 1:117-125.

Brighouse, C. (1997). Determinism and modality. The British Journal for the Philosophy of Science, 48(4):465-481.

Brighouse, C. (2008). Understanding indeterminism. In Dieks, D., editor, The Ontology of Spacetime II, pages 153-173. Elsevier, Oxford.

Butterfield, J. (1989). The hole truth. The British Journal for the Philosophy of Science, 40(1):128.

Christodoulou, D. (2008). Mathematical Problems of General Relativity, volume 1. European Mathematical Society, Zurich.

Dasgupta, S. (2013). Absolutism vs comparativism about quantity. In Bennett, K. and Zimmerman, D. W., editors, Oxford Studies in Metaphysics, volume 8, pages 105-148. Oxford University Press, Oxford.

Dees, M. K. (2015). The fundamental structure of the world: Physical magnitudes, space and time, and the laws of nature. $\mathrm{PhD}$ thesis, Rutgers University.

Dewar, N. (2015). Symmetries and the philosophy of language. Studies in History and Philosophy of Modern Physics, 52:317-327.

Dewar, N. (2016). Symmetries in physics, metaphysics, and logic. PhD thesis, Oxford University.

Dewar, N. (2017). Sophistication about symmetries. The British Journal for the Philosophy of Science, page axx021.

Earman, J. (1986). Why space is not a substance (at least not to first degree). Pacific Philosophical Quarterly, 67:225-44.

Earman, J. (1989). World Enough and Space-Time: Absolute versus Relational Theories of Space and Time. MIT Press, Cambridge, MA.

Earman, J. and Norton, J. (1987). What price spacetime substantivalism? The hole story. The British Journal for the Philosophy of Science, 38(4):515-525.

Eddon, M. (2013). Quantitative properties. Philosophy Compass, 8(7):633-645. 
Feintzeig, B. (2015). On broken symmetries and classical systems. Studies in History and Philosophy of Modern Physics, 52:267 - 273.

Feintzeig, B. (2016). Unitary inequivalence in classical systems. Synthese, 193(9):2685-2705.

Freire, Jr, O. (2015). Quantum Dissidents: Rebuilding the Foundations of Quantum Mechanics (1950-1990). Springer, Berlin.

Frigg, R. and Hartmann, S. (2017). Models in science. In Zalta, E. N., editor, The Stanford Encyclopedia of Philosophy. Metaphysics Research Lab, Stanford University, Spring 2017 edition.

Frigg, R. and Nguyen, J. (2016). Scientific representation. In Zalta, E. N., editor, The Stanford Encyclopedia of Philosophy. Metaphysics Research Lab, Stanford University, Winter 2016 edition.

Iftime, M. and Stachel, J. (2006). The hole argument for covariant theories. General Relativity and Gravitation, 38:1241-1252.

Jammer, M. (1974). The Philosophy of Quantum Mechanics. Wiley, New York.

Lawvere, F. W. and Schanuel, S. H. (2009). Conceptual Mathematics: A First Introduction to Categories. Cambridge University Press, Cambridge, 2nd edition.

Leeds, S. (1995). Holes and determinism: Another look. Philosophy of Science, 62:425-437.

Lehmkuhl, D. (2017). Literal versus careful interpretations of scientific theories: The vacuum approach to the problem of motion in general relativity. Philosophy of Science, 84(5):12021214.

Mac Lane, S. (1998). Categories for the Working Mathematician. Springer, New York, 2nd edition.

Maddy, P. (2007). Second Philosophy: A Naturalistic Method. Oxford University Press, Oxford.

Maudlin, T. (1989). The essence of spacetime. In Fine, A. and Leplin, J., editors, PSA 1988: Proceedings of the Biennial Meeting of the Philosophy of Science Association, volume 2, pages 82-91.

Maudlin, T. (1990). Substances and spacetimes: What aristotle would have said to einstein. Studies in the History and Philosophy of Science, 21(1):531-61.

Melia, J. (1999). Holes, haecceitism and two conceptions of determinism. The British Journal for the Philosophy of Science, 50(4):639-664.

Muller, F. A. (2011). How to defeat Wüthrich's abysmal embarrassment argument against spacetime structuralism. Philosophy of Science, 78(5):10461057.

Mundy, B. (1992). Space-time and isomorphism. PSA: Proceedings of the Biennial Meeting of the Philosophy of Science Association, 1992(1):515-527.

Norton, J. D. (2015). The hole argument. In Zalta, E. N., editor, The Stanford Encyclopedia of Philosophy. Metaphysics Research Lab, Stanford University, Fall 2015 edition. 
O’Neill, B. (1983). Semi-Riemannian Geometry, with Applications to Relativity. Academic Press, San Diego.

Pooley, O. (2013). Substantivalist and relationalist approaches to spacetime. In Batterman, R., editor, The Oxford Handbook of Philosophy of Physics. Oxford University Press, Oxford.

Rickles, D. (2008). Symmetry, Structure, and Spacetime. Elsevier, Amsterdam.

Rosenstock, S., Barrett, T., and Weatherall, J. O. (2015). On Einstein algebras and relativistic spacetimes. Studies in History and Philosophy of Modern Physics, 52:309-16.

Rovelli, C. (2014). Why gauge? Foundations of Physics, 44(1):91-104.

Ruetsche, L. (2011). Interpreting Quantum Theories. Oxford University Press, Oxford.

Rynasiewicz, R. (1992). Rings, holes and substantivalism: On the program of Leibniz algebras. Philosophy of Science, 59:572-89.

Rynasiewicz, R. (1996). Is there a syntactic solution to the hole problem? Philosophy of Science, 63:S55-S62.

Stachel, J. (1989). Einstein's search for general covariance, 1912-1915. In Howard, D. and Stachel, J., editors, Einstein and the History of General Relativity, pages 1-63. Birkhäuser, Boston.

Stachel, J. (2014). The hole argument and some physical and philosophical implications. Living Reviews in Relativity, 17(1):1.

Suárez, M. (2003). Scientific representation: Against similarity and isomorphism. International Studies in the Philosophy of Science, 17:225-244.

Suárez, M. (2004). An inferential conception of scientific representation. Philosophy of Science, 71:767-779.

Weatherall, J. O. (2016a). Fiber bundles, yang-mills theory, and general relativity. Synthese, 193(8):2389-2425.

Weatherall, J. O. (2016b). Regarding the 'hole argument'. The British Journal for the Philosophy of Science, pages n/a-n/a.

Weatherall, J. O. (2016c). Understanding gauge. Philosophy of Science, 85(5):1039-1049.

Wilson, M. (1993). There's a hole and a bucket, dear Leibniz. Midwest Studies In Philosophy, 18(1):202-241.

Wüthrich, C. (2010). Challenging the spacetime structuralist. Philosophy of Science, 76(5):10391051. 